\title{
EFFECTS OF HEAT SOURCE/SINK ON MHD FLOW AND HEAT TRANSFER OVER A SHRINKING SHEET WITH MASS SUCTION
}

\author{
Krishnendu Bhattacharyya* \\ Department of Mathematics, The University of Burdwan, Burdwan-713104, West Bengal, India
}

Received 23 November 2010; accepted 11 August 2011

\begin{abstract}
An analysis is made to study the effects heat source/sink on the steady two dimensional magnetohydrodynamic (MHD) boundary layer flow and heat transfer past a shrinking sheet with wall mass suction. In the dynamic system, a uniform magnetic field acts normal to the plane of flow. The governing partial differential equations are transformed into self-similar ordinary differential equations using similarity transformations. Then the obtained self-similar equations are solved by finite difference method using quasilinearization technique. From the analysis it is found that the velocity inside the boundary layer increases with increase of wall mass suction and magnetic field and accordingly the thickness of the momentum boundary layer decreases. The temperature decreases with Hartmann number, Prandtl number and heat sink parameter and the temperature increases with heat source parameter. Furthermore, for strong heat source heat absorption at the sheet occurs.
\end{abstract}

Keywords: MHD boundary layer, heat transfer, shrinking sheet, heat source/sink, mass suction

DOI:10.3329/cerb.v15i1.6524

\section{Introduction}

The steady boundary layer flow of an incompressible viscous fluid over a stretching sheet has many applications in manufacturing industries and technological processes, such as, glass-fiber production, wire drawing, paper production, metal and polymer processing industries and many others. Crane [1] first considered the steady laminar boundary layer flow of a Newtonian fluid caused by a linearly stretching flat sheet and found an exact similarity solution in closed analytical form. Gupta and Gupta [2] discussed the heat and mass transfer for the Newtonian boundary layer flow over a stretching sheet with suction or blowing. Wang [3] investigated the three-dimensional flow due to the stretching surface. The uniqueness of the solution obtained by Crane [1] was established by McLeod and Rajagopal [4]. The MHD boundary layer theory has a significant contribution in developing of magnetohydrodynamic theory. Due to the important effects of magnetic field on the boundary layer, the study of MHD flow with heat transfer is always interesting. In author's knowledge, the first attempt to study the MHD flow over a stretching surface in an electrically conducting fluid was made by Pavlov [5] in the presence of a uniform transverse magnetic field and he obtained an exact similarity solution. Furthermore, some important contributions in stretching sheet

*Corresponding author Email: krish.math@yahoo.com; krishnendu84@yahoo.com flow were made by Chakrabarti and Gupta [6], Andersson [7], Pop [8] and Bhattacharyya and Layek [9].

Recently, the flow of incompressible fluid due to a shrinking sheet is gaining attention of modern day researchers because of its increasing application to many engineering problems. A steady flow over a shrinking sheet is not possible because the generated vorticity is not confined within the boundary layer. So, to overcome this difficulty the flow needs a certain amount of external opposite force at the sheet. Wang [10] observed the flow around the shrinking sheet while studying the flow behaviour of liquid film over an unsteady stretching sheet. The existence and uniqueness of the solution of steady viscous flow over a shrinking sheet was established by Miklavči $\breve{c}$ and Wang [11]. Hayat et al. [12] reported an analytic solution of MHD flow of a second grade fluid over a shrinking sheet. Muhaimin et al. [13] showed the effects of heat and mass transfer on MHD boundary layer flow past a shrinking sheet subject to suction. Fang and Zhang [14] obtained a closed-form analytical solution for steady MHD flow over a porous shrinking sheet subjected to mass suction. The MHD flow and mass transfer of an upper-convected Maxwell fluid past a porous shrinking sheet with chemically reactive species was studied by Hayat et al. [15]. Fang and Zhang [16] obtained the exact analytic solution of thermal boundary layer over a shrinking sheet with mass transfer. On the other hand, Wang [17] studied the stagnation-point flow towards a shrinking sheet. Wang's [17] work was extended by Ishak et al. [18], Bhattacharyya and 
Layek [19] and Bhattacharyya et al. [20] under different physical conditions.

The study of heat transfer in hydrodynamic boundary layer flow over a porous stretching/shrinking sheet becomes more interesting when internal heat generation or absorption occurs. Effects of heat source or sink on the boundary layer flow over a stretching sheet were studied by Vajravelu and Hadjinicolaou [21], Elbashbeshy and Bazid [22], Bataller [23], Layek et al. [24], Chen [25] and Mahantesh et al. [26].

In the present paper, the effects of heat source or sink on the MHD boundary layer flow and heat transfer over a porous shrinking sheet with mass suction are investigated. Using similarity transformation, the governing partial differential equations are transformed into a set of self-similar non-linear ordinary differential equations, which are then solved numerically by finite difference method using quasilinearization technique. The numerical results are plotted in some figures and the variations in physical characteristics of the flow dynamics and heat transfer for several parameters involved in the equations are discussed in detail.

\section{Formulation of the Problem}

Consider the MHD boundary layer flow of an electrically conducting Newtonian fluid and heat transfer over a permeable shrinking sheet with internal heat generation or absorption. The sheet coincides with the plane $y=0$ and the flow is confined in the region $y>0$. The $x$ and $y$ axes are taken along and perpendicular to the sheet, respectively. A sketch of the physical problem is given in Figure 1. The governing equations of motion for steady two-dimensional flow in presence of uniform transverse magnetic field and the energy equation are written in usual notation as:

$$
\begin{gathered}
\frac{\partial u}{\partial x}+\frac{\partial v}{\partial y}=0 \\
u \frac{\partial u}{\partial x}+v \frac{\partial u}{\partial y}=v \frac{\partial^{2} u}{\partial y^{2}}-\frac{\sigma B_{0}^{2}}{\rho} u \\
u \frac{\partial T}{\partial x}+v \frac{\partial T}{\partial y}=\frac{\kappa}{\rho c_{p}} \frac{\partial^{2} T}{\partial y^{2}}+\frac{Q_{0}}{\rho c_{p}}\left(T-T_{\infty}\right)
\end{gathered}
$$

where $u$ and $v$ are velocity components in $x$ - and $y$ directions respectively, $v(=\mu / \rho)$ is the kinematic fluid viscosity, $\rho$ is the fluid density, $\mu$ is the coefficient of fluid viscosity, $\sigma$ is the electrical conductivity of the fluid, $B_{0}$ is the applied uniform magnetic field, $T$ is the temperature, $T_{\infty}$ is the free stream temperature, $\kappa$ is the fluid thermal conductivity, $c_{p}$ is the specific heat and $Q_{0}$ is the volumetric rate of heat generation or absorption.

The appropriate boundary conditions for the velocity components and temperature are given by:

$u=U_{w}(x)=-c x, v=-v_{w}$ at $y=0 ; u \rightarrow 0$ as $y \rightarrow \infty$

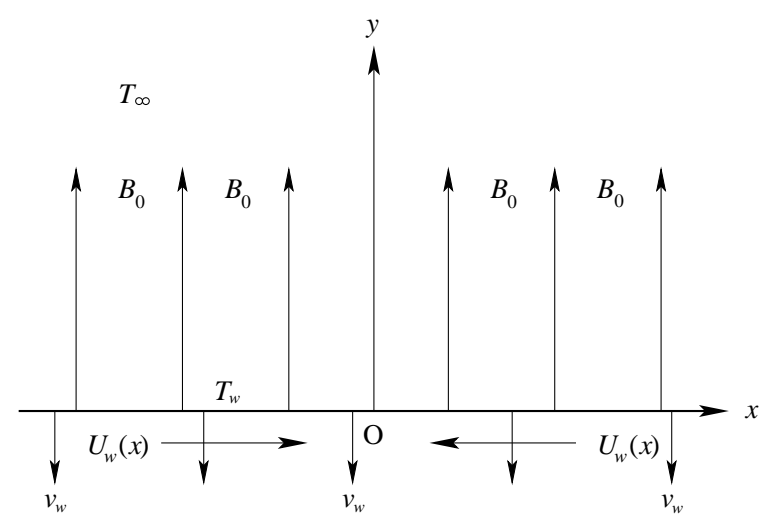

Figure 1: A sketch of the physical problem

$$
T=T_{w} \text { at } y=0 ; T \rightarrow T_{\infty} \text { as } y \rightarrow \infty
$$

where $c>0$ is the shrinking constant and $T_{w}$ is temperature of the sheet. Here $v_{w}(>0)$ is a prescribed distribution of wall mass suction through the porous sheet.

Introducing the stream function $\psi$, the velocity components $u$ and $v$ can be written as:

$$
u=\partial \psi / \partial y \text { and } v=-\partial \psi / \partial x
$$

For relations of (6), the mass-conservation equation (1) is satisfied automatically and the momentum equation (2) and temperature equation (3) take the following forms:

$$
\begin{array}{r}
\frac{\partial \psi}{\partial y} \frac{\partial^{2} \psi}{\partial x \partial y}-\frac{\partial \psi}{\partial x} \frac{\partial^{2} \psi}{\partial y^{2}}=v \frac{\partial^{3} \psi}{\partial y^{3}}-\frac{\sigma B_{0}^{2}}{\rho} \frac{\partial \psi}{\partial y} \\
\frac{\partial \psi}{\partial y} \frac{\partial T}{\partial x}-\frac{\partial \psi}{\partial x} \frac{\partial T}{\partial y}=\frac{\kappa}{\rho c_{p}} \frac{\partial^{2} T}{\partial y^{2}}+\frac{Q_{0}}{\rho c_{p}}\left(T-T_{\infty}\right)
\end{array}
$$

Also the boundary conditions in (4) reduce to:

$$
\frac{\partial \psi}{\partial y}=-c x, \frac{\partial \psi}{\partial x}=v_{w} \text { at } y=0 ; \frac{\partial \psi}{\partial y} \rightarrow 0 \text { as } y \rightarrow \infty
$$

Next, we introduce the dimensionless variables for $\psi$ and $T$ as:

$$
\psi=\sqrt{c v} x f(\eta) \text { and } T=T_{\infty}+\left(T_{w}-T_{\infty}\right) \theta(\eta)
$$

where $\eta$ is the similarity variable and is given by $\eta=$ $y(c / v)^{1 / 2}$.

Using the dimensionless variables in (10) and similarity variable, the above equations (7) and (8) finally have taken the following self-similar forms:

$$
\begin{gathered}
f^{\prime \prime \prime}+f f^{\prime \prime}-f^{\prime 2}-M^{2} f^{\prime}=0 \\
\theta^{\prime \prime}+\operatorname{Pr}\left(f \theta^{\prime}-\lambda \theta\right)=0
\end{gathered}
$$

where $M=\left(\sigma B_{0}^{2} / c \rho\right)^{1 / 2}$ is the Hartmann number, $\operatorname{Pr}=\mu c_{p} / \kappa$ is the Prandtl number and $\lambda=Q_{0} / \rho C_{p} c$ is the heat source $(\lambda<0)$ or sink $(\lambda>0)$ parameter. 
The boundary conditions (9) and (5) also reduce to

$$
\begin{gathered}
f(\eta)=S, f^{\prime}(\eta)=-1 \text { at } \eta=0 ; f^{\prime}(\eta) \rightarrow 0 \text { as } \eta \rightarrow \infty \\
\theta(\eta)=1 \text { at } \eta=0 ; \theta(\eta) \rightarrow 0 \text { as } \eta \rightarrow \infty
\end{gathered}
$$

where $S=v_{w} /(c v)^{1 / 2}(>0)$ is the mass suction parameter.

\section{Numerical Solution}

The nonlinear system of equations (11) and (12) along with the boundary conditions have been solved numerically by finite difference method using the quasilinearization technique [27].

The discretised version of (11) and (12) with the boundary conditions (13) and (14) are written as:

$$
\begin{gathered}
F^{\prime \prime(i+1)}+f^{(i)} F^{(i+1)}-\left(2 F^{(i)}+M^{2}\right) F^{(i+1)}=-F^{2(i)} \\
\theta^{\prime \prime(i+1)}+\operatorname{Pr} f^{(i+1)} \theta^{\prime(i+1)}-\operatorname{Pr} \lambda \theta^{(i+1)}=0
\end{gathered}
$$

where $F=f^{\prime}$.

The boundary conditions become:

$$
\begin{gathered}
f^{(i+1)}=S, F^{(i+1)}=-1 \text { at } \eta=0 ; F^{(i+1)}=0 \text { at } \eta=\eta * \\
\theta^{(i+1)}=1 \text { at } \eta=0 ; \theta^{(i+1)}=0 \text { at } \eta=\eta *
\end{gathered}
$$

The functions with iteration index $(i)$ denote the $i$ th iteration level and the corresponding index $(i+1)$ be the $(i+1)$-th level and $\eta *$ is suitable distance from the origin selected by considering the flow behaviour in the boundary layer region.

We divide the interval $[0, \eta *]$ into $N$ equal subintervals of length $\Delta \eta=0.001$ taking the non-dimensional distance $\eta *=50$ for all cases under investigation. Applying central finite difference formulae of the second and first orders derivatives of $F$ as:

$$
F^{\prime \prime}=\frac{F_{j+1}-2 F_{j}+F_{j-1}}{(\Delta \eta)^{2}} \text { and } F^{\prime}=\frac{F_{j+1}-F_{j-1}}{2(\Delta \eta)}
$$

and similar for $\theta$, the above system of equations (15) and (16) along with the boundary conditions (17) and (18) reduce to:

$$
\begin{gathered}
F_{j-1}^{(i+1)} a_{j}+F_{j}^{(i+1)} b_{j}+F_{j+1}^{(i+1)} c_{j}=d_{j}, 1 \leqslant j \leqslant N \\
\theta_{j-1}^{(i+1)} p_{j}+\theta_{j}^{(i+1)} q_{j}+\theta_{j+1}^{(i+1)} r_{j}=0,1 \leqslant j \leqslant N \\
\text { with } f_{0}^{(i+1)}=S, F_{0}^{(i+1)}=-1 \text { and } F_{N+1}^{(i+1)}=0 \\
\text { and } \theta_{0}^{(i+1)}=1 \text { and } \theta_{N+1}^{(i+1)}=0
\end{gathered}
$$

where $a_{j}=\frac{1}{(\Delta \eta)^{2}}-\frac{f_{j}^{(i)}}{2(\Delta \eta)}, b_{j}=-\frac{2}{(\Delta \eta)^{2}}-2 F_{j}^{(i)}-M^{2}$, $c_{j}=\frac{1}{(\Delta \eta)^{2}}+\frac{f_{j}^{(i)}}{2(\Delta \eta)}, d_{j}=-F_{j}^{2(i)}, p_{j}=\frac{1}{(\Delta \eta)^{2}}-\frac{\operatorname{Pr} f_{j}^{(i+1)}}{2(\Delta \eta)}$, $q_{j}=-\frac{2}{(\Delta \eta)^{2}}-\operatorname{Pr} \lambda, r_{j}=\frac{1}{(\Delta \eta)^{2}}+\frac{\operatorname{Pr} f_{j}^{(i+1)}}{2(\Delta \eta)} ; 1 \leqslant j \leqslant N$.

We solve the system of algebraic (tri-diagonal system) equations (19) with the conditions (21) by the standard Thomas algorithm. Using the newly obtained results for $f_{j}^{(i+1)}$ and $F_{j}^{(i+1)}$ the system (20), the discretised temperature equation with the conditions (22) is then solved by the same Thomas algorithm.

Table 1: Skin friction coefficient $f^{\prime \prime}(0)$ for different values of $S$ with
\begin{tabular}{crc}
$M^{2}=2$ & \\
\hline$S$ & Present study & Muhaimin et al. [13] \\
\hline 2 & 2.414300 & 2.414214 \\
3 & 3.302750 & 3.302776 \\
4 & 4.236099 & 4.236068 \\
\hline
\end{tabular}

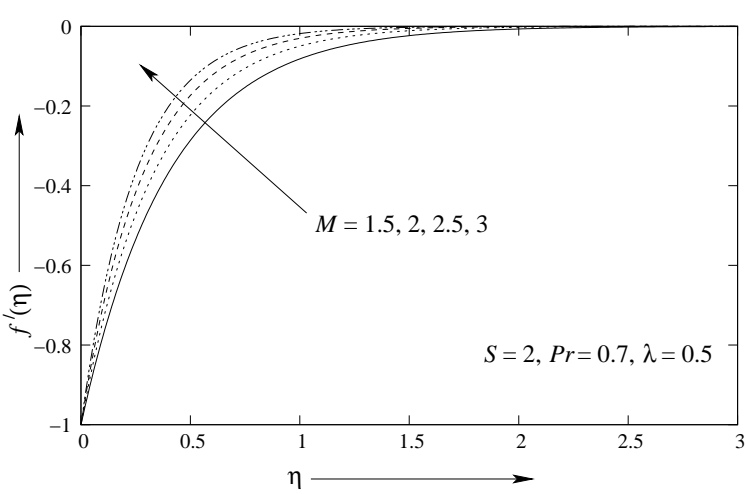

Figure 2: Velocity profiles $f^{\prime}(\eta)$ for several values of $M$

\section{Results and Discussion}

Numerical computations are performed for various values of the physical parameters involved in the equation viz. the Hartmann number $M$, the mass suction parameter $S$, the Prandtl number $\mathrm{Pr}$ and the heat source/sink parameter $\lambda$. To ensure the occurrence of steady flow near the sheet by confining the generated vorticity inside the boundary layer, the opposite forces i.e. the magnetic field and the wall mass suction are taken quite strong by assigning large values of $M$ and $S$. The calculated results are presented in Figures 2-8 to understand the effects of parameters on the flow and temperature field.

In order to assure the accuracy of the applied numerical scheme the computed values of skin friction coefficient $f^{\prime \prime}(0)$ are compared with the available results of Muhaimin et al. [13] in Table 1 and have found in excellent agreement.

The impacts of the Hartman number $M$ on the velocity and temperature profiles are very significant in practical point of view. In Figure 2 and Figure 3, the variations in velocity field and temperature distribution for several values of $M$ are presented. The dimensionless velocity $f^{\prime}(\eta)$ increases with increasing values of $M$. Accordingly, the thickness of the momentum boundary layer decreases. This happens due to the Lorentz force arising from the interaction of magnetic and electric fields during the motion of the electrically conducting fluid. To reduce momentum boundary layer thickness the generated Lorentz force enhances the fluid motion in the boundary layer region. On the other hand, from Figure 3, it is noticed that the temperature $\theta(\eta)$ at a point decreases with $M$.

Now we see the effects of mass suction parameter $S$ on the velocity and temperature profiles. The veloc- 


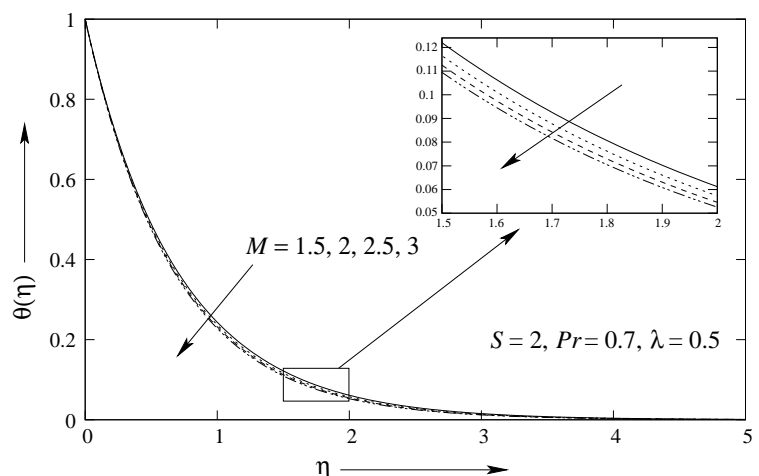

Figure 3: Temperature profiles $\theta(\eta)$ for several values of $M$

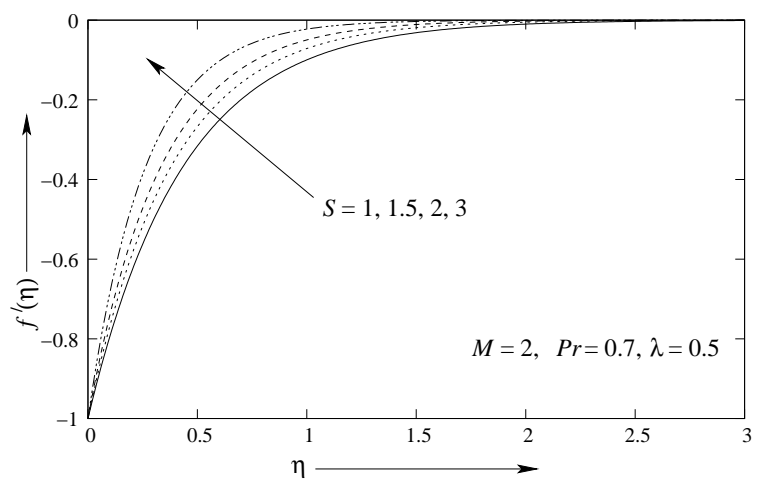

Figure 4: Velocity profiles $f^{\prime}(\eta)$ for several values of $S$

ity profiles for various values of suction parameter $S$ are depicted in Figure 4. From the figure it is noted that for a fixed value of $\eta$, velocity profiles increase as applied suction increases and it makes the momentum boundary layer thickness thinner. The dimensionless temperature profiles for several values of suction parameter are demonstrated in Figure 5. It is seen that the wall mass suction affects the temperature distribution in addition to the velocity field. With increasing suction the temperature $\theta(\eta)$ for fixed $\eta$ decreases and consequently, the thermal boundary layer thickness reduces.

The temperature field for various values of the Prandtl number $\mathrm{Pr}$ is represented in Figure 6. With increasing $P r$, the dimensionless temperature profile as well as thermal boundary layer thickness quickly decrease. An increase in Prandtl number means a decrease of fluid thermal conductivity which causes a decrease in temperature. Since the momentum equation is independent of $\theta$, so no effect of $\operatorname{Pr}$ on the velocity field is observed.

In Figure 7, the effect heat source or sink parameter $\lambda$ on the temperature is exhibited. From the figure it is noticed that the dimensionless temperature $\theta(\eta)$ decreases for increasing strength of the heat sink and due to increase of heat source strength the temperature increases. So, the thickness of thermal boundary

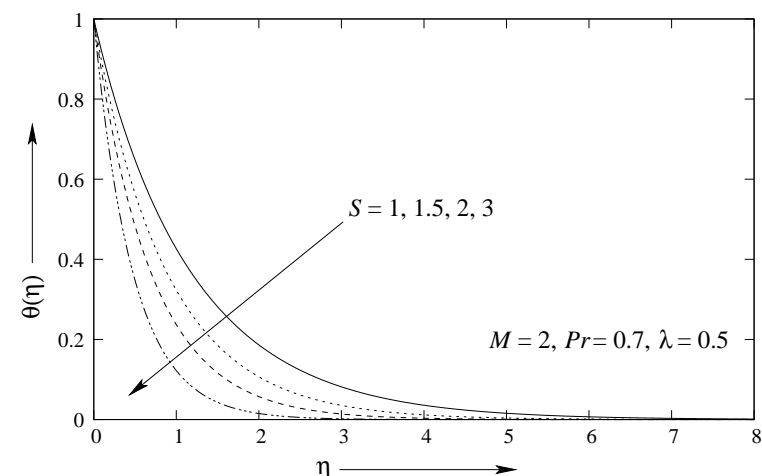

Figure 5: Temperature profiles $\theta(\eta)$ for several values of $S$

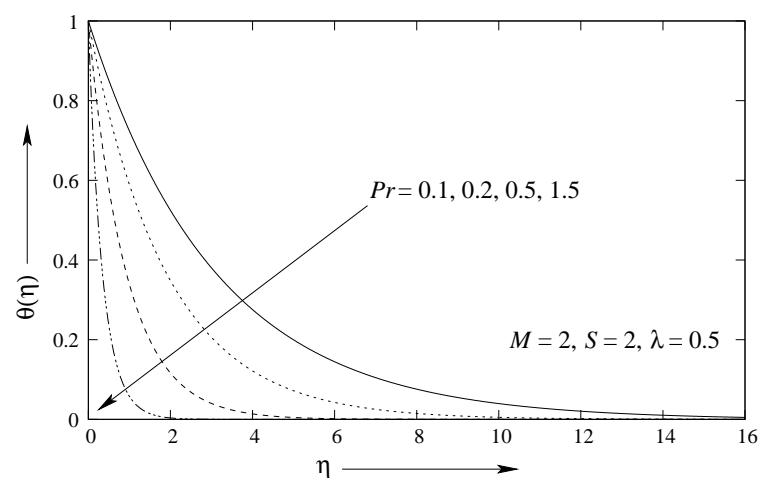

Figure 6: Temperature profiles $\theta(\eta)$ for several values of $\mathrm{Pr}$

layer reduces for increase of heat sink parameter, but it increases with heat source parameter. This result is very much significant for the flow where heat transfer is given prime importance.

The variation of the temperature gradient at the sheet $\theta^{\prime}(0)$ which is a significant in evaluating the rate of heat transfer from the sheet is presented in Figure 8 for various values of $\operatorname{Pr}$ and $\lambda$. The negative value of $\theta^{\prime}(0)$ means heat transfer and the positive value means heat absorption. The rate of heat transfer increases with Prandtl number. While, an interesting nature is found for the variation of heat source/ sink parameter. When the Prandtl number is small, for large values of heat source parameter $(\lambda<0)$ heat absorption occurs at the sheet. Although, for increase of heat sink parameter $(\lambda>0)$ heat transfer increases.

\section{Conclusion}

The effects of heat source/sink on the MHD boundary layer flow and heat transfer over a shrinking sheet subject to strong suction is investigated. The selfsimilar equations are obtained using similarity transformations. The self-similar equations are linearised by the quasilinearization technique and are then solved by finite difference method. The study reveals that due to increase of the Hartmann number and the mass suc- 


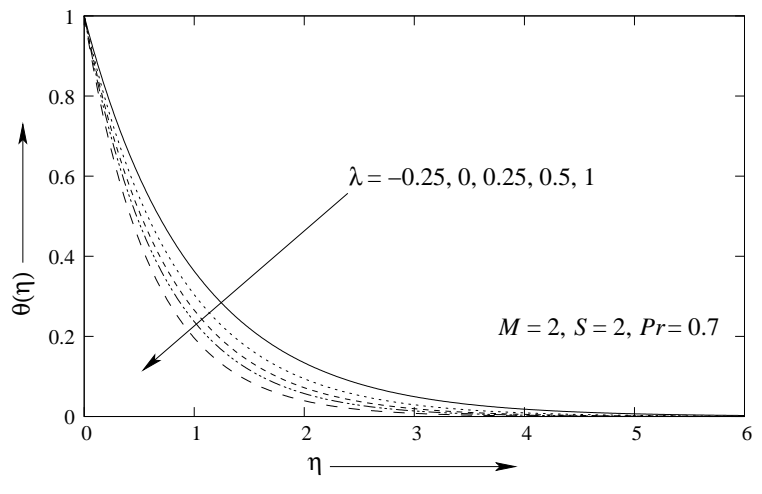

Figure 7: Temperature profiles $\theta(\eta)$ for several values of $\lambda$

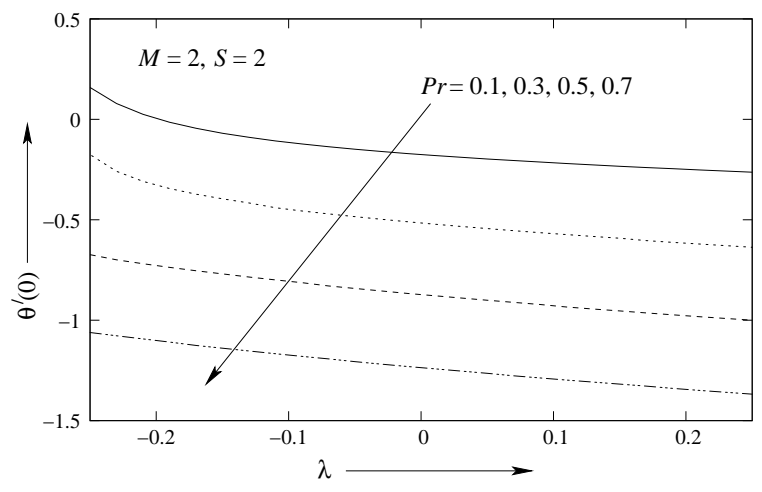

Figure 8: Temperature gradient at the sheet $\theta^{\prime}(0)$ against $\lambda$ for several values of $\mathrm{Pr}$

tion parameter the momentum boundary layer thickness reduces and the temperature at a point decreases. The temperature as well as the thermal boundary layer thickness decrease with increasing values of Prandtl number. Most importantly, for some higher values of heat source parameter heat absorption occurs at the sheet. The heat transfer is enhanced for increase of Prandtl number and heat sink parameter, this heat transfer is very important in production engineering to improve the quality of the final product.

\section{References}

[1] Crane LJ, Flow past a stretching plate, Zeitschrift für Angewandte Mathematik und Physik, 1970. 21(4):pp. 645-647

[2] Gupta PS and Gupta AS, Heat and mass transfer on a stretching sheet with suction or blowing, The Canadian Journal of Chemical Engineering, 1977. 55(6):pp. 744-746

[3] Wang CY, The three-dimensional flow due to a stretching flat surface, Physics of Fluids, 1984. 27:pp. 1915-1917

[4] McLeod JB and Rajagopal KR, On the uniqueness of flow of a Navier-Stokes fluid due to a stretching boundary, Archive for Rational Mechanics and Analysis, 1987. 98(4):pp. 385-393

[5] Pavlov KB, Magnetohydrodynamic flow of an incompressible viscous fluid caused by deformation of a plane surface, Magnitnaya Gidrodinamika, 1974. 4:pp. 146-147

[6] Chakrabarti A and Gupta AS, Hydromagnetic flow and heat transfer over a stretching sheet, Quarterly Journal of Mechanics and Applied Mathematics, 1979. 37:pp. 73-78

[7] Andersson HI, MHD flow of a viscoelastic fluid past a stretching surface, Acta Mechanica, 1992. 95(1):pp. 227-230
[8] Dirks CA, Gouverneur M, McCullum L, McGovern C, Melsness J, Saunders S, Pop I and Na TY, A note on MHD flow over a stretching permeable surface, Mechanics Research Communications, 1998. 25(3):pp. 263-269

[9] Bhattacharyya $\mathrm{K}$ and Layek GC, Chemically reactive solute distribution in MHD boundary layer flow over a permeable stretching sheet with suction or blowing, Chemical Engineering Communications, 2010. 197(12):pp. 1527-1540

[10] Wang CY, Liquid film on an unsteady stretching sheet, Quarterly of Applied Mathematics, 1990. 48(4):pp. 601-610

[11] Miklavči $\breve{c}$ M and Wang CY, Viscous flow due to a shrinking sheet, Quarterly of Applied Mathematics, 2006. 64(2):pp. 283-290

[12] Hayat T, Abbas Z and Sajid M, On the analytic solution of magnetohydrodynamic flow of a second grade fluid over a shrinking sheet, Journal of Applied Mechanics, 2007. 74:pp. 1165-1171

[13] Kandasamy R and Khamis AB, Effects of heat and mass transfer on nonlinear MHD boundary layer flow over a shrinking sheet in the presence of suction, Applied Mathematics and Mechanics, 2008. 29(10):pp. 1309-1317

[14] Fang T and Zhang J, Closed-form exact solutions of MHD viscous flow over a shrinking sheet, Communications in Nonlinear Science and Numerical Simulation, 2009. 14(7):pp. 28532857

[15] Hayat T, Abbas Z and Ali N, MHD flow and mass transfer of a upper-convected Maxwell fluid past a porous shrinking sheet with chemical reaction species, Physics Letters A, 2008. 372(26):pp. 4698-4704

[16] Fang T and Zhang J, Thermal boundary layers over a shrinking sheet: an analytical solution, Acta Mechanica, 2010. 209(3):pp. 325-343

[17] Wang CY, Stagnation flow towards a shrinking sheet, International Journal of Non-Linear Mechanics, 2008. 43(5):pp. 377382

[18] Ishak A, Lok Y and Pop I, Stagnation-point flow over a shrinking sheet in a micropolar fluid, Chemical Engineering Communications, 2010. 197(11):pp. 1417-1427

[19] Bhattacharyya K and Layek G, Effects of suction/blowing on steady boundary layer stagnation-point flow and heat transfer towards a shrinking sheet with thermal radiation, International Journal of Heat and Mass Transfer, 2011. 54:pp. 302307

[20] Bhattacharyya K, Mukhopadhyay S and Layek G, Slip effects on boundary layer stagnation-point flow and heat transfer towards a shrinking sheet, International Journal of Heat and Mass Transfer, 2011. 54:pp. 308-313

[21] Vajravelu K and Hadjinicolaou A, Heat transfer in a viscous fluid over a stretching sheet with viscous dissipation and internal heat generation, International Communications in Heat and Mass Transfer, 1993. 20(3):pp. 417-430

[22] Elbashbeshy EMA and Bazid MAA, Heat transfer in a porous medium over a stretching surface with internal heat generation and suction or injection, Applied mathematics and computation, 2004. 158(3):pp. 799-807

[23] Bataller RC, Effects of heat source/sink, radiation and work done by deformation on flow and heat transfer of a viscoelastic fluid over a stretching sheet, Computers \& Mathematics with Applications, 2007. 53(2):pp. 305-316

[24] Layek GC, Mukhopadhyay S and Samad SA, Heat and mass transfer analysis for boundary layer stagnation-point flow towards a heated porous stretching sheet with heat absorption/generation and suction/blowing, International communications in heat and mass transfer, 2007. 34(3):pp. 347-356

[25] Chen CH, Magneto-hydrodynamic mixed convection of a power-law fluid past a stretching surface in the presence of thermal radiation and internal heat generation/absorption, International Journal of Non-Linear Mechanics, 2009. 44(6):pp. 596-603

[26] Nandeppanavar MM, Abel MS and Tawade J, Heat transfer in a Walter's liquid B fluid over an impermeable stretching 
sheet with non-uniform heat source/sink and elastic deformation, Communications in Nonlinear Science and Numerical Simulation, 2010. 15(7):pp. 1791-1802

[27] Bellman RE and Kalaba RE, Quasilinearization and nonlinear boundary-value problems, American Elsevier Pub. Co., New York, 1965 